\title{
Coherent silicon photonic interferometric biosensor with an inexpensive laser source for sensitive label-free immunoassays
}

\author{
Jonas Leuermann, ${ }^{1,2, *}$ (i) Vladimir Stamenkovic, ${ }^{1,3}$ Patricia Ramirez-Priego, ${ }^{4}$ \\ Alejandro Sánchez-Postigo, ${ }^{2}$ (i) Adrián Fernández-Gavela, ${ }^{5}$ Cole A. Chapman, ${ }^{6}$ \\ Ryan C. Bailey, ${ }^{6}$ Laura M. Lechuga, ${ }^{4}$ (D) Ezequiel Perez-Inestrosa, ${ }^{1,3}$ \\ Daniel Collado, ${ }^{1,3}$ Robert Halir, ${ }^{1,2}$ and Í̃̃̃go Molina-Fernández ${ }^{1,2}$ (i) \\ ${ }^{1}$ Bionand Center for Nanomedicine and Biotechnology, Parque Tecnológico de Andalucía, 29590 Málaga, Spain \\ ${ }^{2}$ Departamento de Ingeniería de Comunicaciones, University of Málaga, ETSI Telecomunicación, Campus de Teatinos, 29071 Málaga, Spain \\ ${ }^{3}$ Departmento de Química Orgánica, Facultad de Ciencias, University of Málaga, 29071 Málaga, Spain \\ ${ }^{4}$ Nanobiosensors and Bioanalytical Applications Group, Catalan Institute of Nanoscience and Nanotechnology (ICN2), CSIC, \\ BIST and CIBER-BBN Campus UAB, 08193 Barcelona, Spain \\ ${ }^{5}$ Departamento de Física, Universidad de Oviedo, C/Federico García Lorca, 33007 Oviedo, Spain \\ ${ }^{6}$ Department of Chemistry, University of Michigan, 930 North University Avenue, Ann Arbor, Michigan 48109, USA \\ ${ }^{*}$ Corresponding author: jonas.leuermann@uma.es
}

Received 2 October 2020; revised 7 November 2020; accepted 9 November 2020; posted 10 November 2020 (Doc. ID 411635); published 8 December 2020

\begin{abstract}
Over the past two decades, integrated photonic sensors have been of major interest to the optical biosensor community due to their capability to detect low concentrations of molecules with label-free operation. Among these, interferometric sensors can be read-out with simple, fixed-wavelength laser sources and offer excellent detection limits but can suffer from sensitivity fading when not tuned to their quadrature point. Recently, coherently detected sensors were demonstrated as an attractive alternative to overcome this limitation. Here we show, for the first time, to the best of our knowledge, that this coherent scheme provides sub-nanogram per milliliter limits of detection in C-reactive protein immunoassays and that quasi-balanced optical arm lengths enable operation with inexpensive Fabry-Perot-type lasers sources at telecom wavelengths. () 2020 Optical Society of America under the terms of the OSA Open Access Publishing Agreement
\end{abstract}

https://doi.org/10.1364/OL.411635

Integrated photonic biosensors have been the subject of intense research in the last two decades, due not only to their miniturizability but also because they can quantitatively detect extremely low concentrations of analytes in real time and without the need of labeling tags [1-3]. Silicon-based sensors provide particularly compact footprints, enabling multiplexed detection of different biochemical substances, and CMOS compatible fabrication, which paves the way towards low-cost mass production [4]. Therefore, they are candidates for point-of-care (POC) solutions in which pre-functionalized, disposable chips are used in conjunction with a read-out system to provide diagnostics that would otherwise require specialized laboratories and trained personnel [5]. Most photonic integrated sensors rely on the principle of evanescent field sensing: the analyte attaches to a biorecognition layer on the waveguide surface, where it interacts with the evanescent field of the guided wave, changing its phase [6-8]. Since changes in the optical phase cannot be measured directly, they are converted into changes in optical power, using on-chip resonant or interferometric structures.

Resonant structures convert the phase change into a resonance wavelength shift, thus often requiring a read-out system with a tunable external cavity laser (ECL) [9-14], or a broadband light source combined with additional wavelength filtering [15]. For homogeneous refractive index sensing, these systems offer moderate limits of detection (LODs), on the order of $10^{-6}$ refractive index units (RIUs), but can achieve very compact footprints $[9,16,17]$. Regarding the detection of biomolecules, in benchmark primary C-reactive protein (CRP) binding assays, resonant sensors achieve LODs on the order of $10 \mathrm{ng} / \mathrm{mL}(0.4 \mathrm{nM})$ [18]. Interferometric sensors compare the optical wave in the sensing waveguide to a wave in a reference waveguide to reconstruct the phase shift. These sensors generally operate with a fixed-spectrum laser source, which can be a superluminescent diode, with asymmetric sensing and reference arm and optical wavelength filtering in the read-out $[2,19,20]$, or a narrow-linewidth laser source, e.g., a $\mathrm{He}-\mathrm{Ne}$ laser at visible wavelengths [21-23] or ECLs at telecom wavelengths $[10,24,25]$. Detection limits down to $2 \mathrm{ng} / \mathrm{mL}$ $(84 \mathrm{pM})$ with a label-free primary binding immunoassay for detecting CRP have been reported for Mach-Zehnder interferometers (MZIs) [16,26]. It is noteworthy that shortcomings commonly associated with these interferometric sensors, such as sensitivity fading when not operated at the quadrature point and phase ambiguity, have been overcome, either by adding a 
(a)

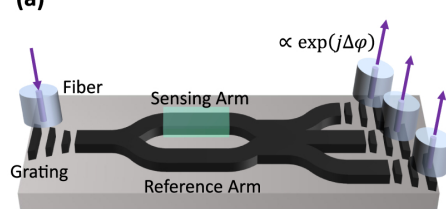

(b)
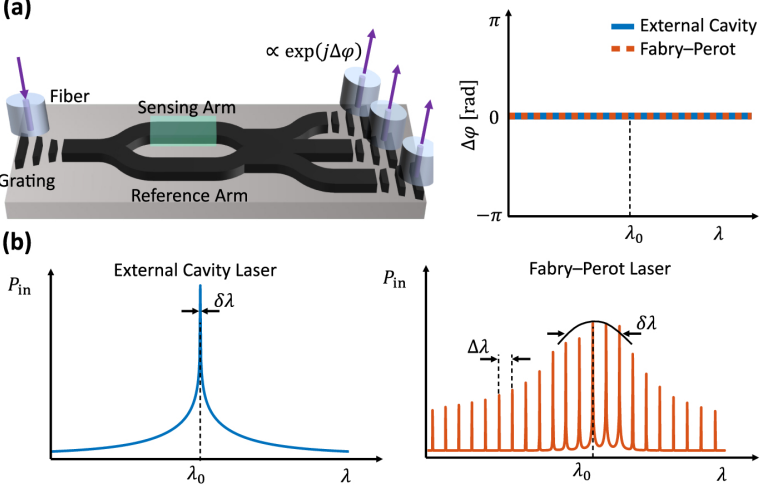

Fig. 1. (a) Schematic of a coherently read balanced Mach-Zehnder sensor, where the phase difference between the reference and sensing arms is recovered from three output signals. The wavelength response of such a sensor is completely flat when the optical arm lengths are balanced. (b) Approximated laser spectra of a narrow-linewidth external cavity laser and a Fabry-Perot laser, with center wavelength $\lambda_{0}$, linewidth $\delta \lambda$, and spectral spacing $\Delta \lambda$.

modulation signal [27-29] or by using a coherent detection approach, illustrated in Fig. 1(a) [24,30-34]. With coherent detection, no tuning to a quadrature point is required, so that the optical lengths of the interferometer arms can be perfectly matched. So far, this approach has been demonstrated only with bulk sensing experiments [10,24], and using high-quality ECLs with narrow linewidths [see Fig. 1(b)]. Here we show, for the first time, that this coherent approach is well suited for protein detection, reporting a limit of detection better than $300 \mathrm{pg} / \mathrm{mL}$ for primary binding CRP detection. We furthermore show that due to quasi-identical optical arm lengths, the sensor can operate with a low-cost Fabry-Perot telecom laser [Fig. 1(b)] with an acceptable performance penalty.

Mach-Zehnder-based sensors split the incoming light into two single-mode waveguides, one acting as the sensing arm and the other acting as the reference arm [Fig. 1(a)]. The reference waveguide is buried in silicon dioxide, so that the propagating mode always experiences a constant phase shift. In sensing waveguides, the propagating mode interacts with the analyte, which causes an additional phase shift. Conventional MZIs recombine these two arms into one output waveguide (e.g., using a Y-Junction or a $1 \times 2$ multi-mode interference coupler). This results in a signal proportional to $\sin (\Delta \varphi)$, where $\Delta \varphi$ is the phase difference between the waves in the two arms. This signal is affected by relative intensity noise, and causes sensitivity fading and phase ambiguity [16]. In contrast, a coherently read MZI recombines the sensing and reference arms via a $2 \times 3$ coupler [Fig. 1(a)]. From the three output powers, a complex signal $\propto \exp (j \Delta \varphi)$ can be calculated [31,35], with the extracted phase being immune to relative intensity noise, sensitivity fading, and phase ambiguity [34]. The noise in the extracted phase caused by a finite laser linewidth $\delta f$ is given by $2 \Delta \tau \sqrt{\pi \delta f B}$, where $B$ is the measurement bandwidth, and $\Delta \tau$ is the time delay between the reference and sensing arms, $\Delta \tau=\left|L_{\mathrm{s}} n_{\mathrm{g}, \mathrm{s}}-L_{\mathrm{r}} n_{\mathrm{g}, \mathrm{r}}\right| / c_{0}$, with $L_{\mathrm{s}}, L_{\mathrm{r}}$ the physical lengths of the sensing and reference arms, $n_{\mathrm{g}, \mathrm{s}}, n_{\mathrm{g}, \mathrm{r}}$ the group indices, and $c_{0}$ the speed of light [34]. Thus, by choosing $L_{s}$ and $L_{\mathrm{r}}$ such that $\Delta \tau=0$, the phase noise can be minimized. Intuitively, this can be understood because for a perfectly balanced interferometer, the wavelength response is flat [Fig. 1(a)].
In our experiments, the reference and sensing arms have the same length $L=8.8 \mathrm{~mm}$ and a group index difference of $\Delta n_{\mathrm{g}, \mathrm{rs}}=\left|n_{\mathrm{g}, \mathrm{s}}-n_{\mathrm{g}, \mathrm{r}}\right| \approx 0.01$ at $\lambda_{0}=1.55 \mu \mathrm{m}$.

In our read-out system, the light source is connected to an optional erbium-doped fiber amplifier and a variable optical attenuator to obtain the desired input power. A polarization rotator is used to achieve TE polarization that is coupled into the silicon nitride sensor chip via grating couplers, with coupling losses of $\sim 10 \mathrm{~dB}$ [Fig. 1(a)]. The sensor chip is fabricated with a multi-project wafer approach in a $300 \mathrm{~nm}$ thick silicon nitride film [36]. The sensing arm is covered with a polydimethylsiloxane (PDMS) microfluidic channel, with a width of $1 \mathrm{~mm}$, length of $9.8 \mathrm{~mm}$, and height of $0.5 \mathrm{~mm}$, which is connected to a syringe pump. In between, a six-port valve system enables the injection of an analyte volume of $V_{\text {inj }} \approx 120 \mu \mathrm{L}$ into the channel. The three optical output signals are coupled to a fiber array using on-chip identical grating couplers and are detected by separate photodiodes (PDs) with integrated transimpedance amplifiers. The resulting voltages are quantized by a data acquisition board, and signal processing is used to estimate the phase change $\Delta \varphi$ [31]. A detailed description and bulk LOD optimization of the experimental setup can be found in [24]. To assess the impact of the laser linewidth on sensor performance, two different lights sources were employed in the experiments. As a high-quality reference, we used the WSL-100 from SANTEC (Japan), a single-mode ECL bought for approximately $10,000 €$, with a linewidth of $\delta \lambda<80 \mathrm{fm}$ $(100 \mathrm{kHz})$ [Fig. 1(b)]. As a low-cost alternative, we used a hand-held Fabry-Perot laser (FPL) with a center wavelength of $\lambda_{0}=1.543 \mu \mathrm{m}, \Delta \lambda=1.36 \mathrm{~nm}$ wavelength spacing, and a bandwidth of $\delta \lambda \sim 3.6 \mathrm{~nm}(450 \mathrm{GHz})$ [Fig. 1(b)], purchased on Amazon for $34 €$.

Two types of experiments were chosen to determine the quality of the sensor, bulk refractive index sensing, and protein CRP immunodetection. We used two different power levels: low, with a total optical power of approximately of $-30 \mathrm{dBm}$ received by the PDs, and high, with a power of approximately $-10 \mathrm{dBm}$. Homogeneous refractive index sensing experiments were performed by injecting four different mass percentage solutions of sodium chloride $(3 \%, 6 \%, 9 \%$, $12 \%)$ resulting in changes of $(0.5,1.0,1.5,2.0) \times 10^{-2} \mathrm{RIU}$

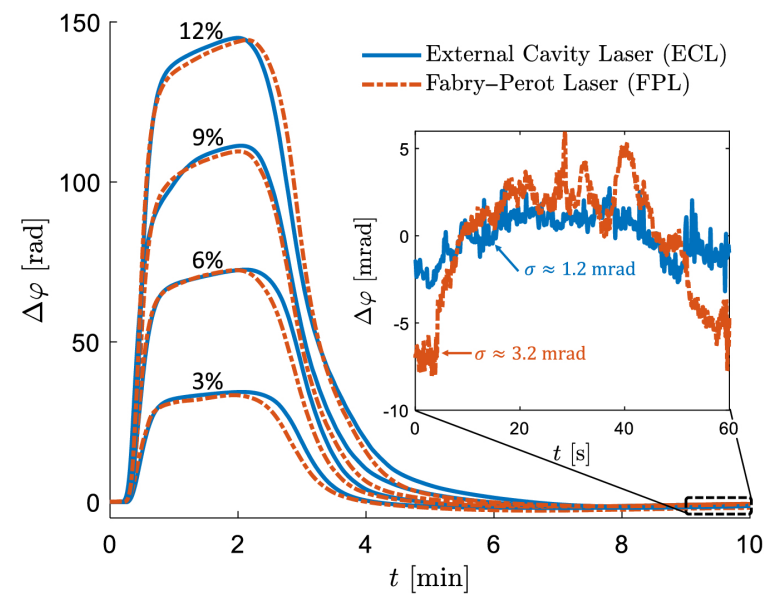

Fig. 2. Bulk sensing using the reference ECL source (continuous blue curve) and the low-cost FPL (dashed orange curve) for 3\%, 6\%, $9 \%$, and $12 \%$ mass percentage sodium chloride injections and low input power. 
Table 1. Detection Limits in RIU for All Homogeneous Refractive Index Sensing Experiments, Categorized by Optical Source and Power Level ${ }^{a}$

\begin{tabular}{lcc}
\hline & Low Power & High Power \\
\hline ECL & $5 \times 10^{-7}$ & $9 \times 10^{-8}$ \\
FPL & $1 \times 10^{-6}$ & $5 \times 10^{-7}$ \\
\hline
\end{tabular}

${ }^{a}$ Each LOD represents the average value of three experiments.

compared to the purified water (Milli-Q) flowing buffer [37]. A flow velocity of $F=30 \mu \mathrm{L} /$ min was maintained throughout the experiments. An example of the induced phase shifts due to the four different sodium chloride concentrations evaluated with low power by both light sources is shown in Fig. 2 . The blue solid and the orange dashed curves correspond to the ECL and the FPL source, respectively. Both are highly correlated and produce a similar total phase response. The mean extracted sensitivity is $S \sim 7100 \mathrm{rad} / \mathrm{RIU}$ which is close to the theoretical value of $S=(2 \pi / \lambda) S_{\mathrm{WG}} L_{\mathrm{s}} \approx 7800 \mathrm{rad} / \mathrm{RIU}$, with $S_{\text {WG }}$ the waveguide sensitivity, following the definition in [34]. The inset shows a $60 \mathrm{~s}$ fragment of the read-out noise, and the corresponding standard deviation $(\sigma)$. These experiments were carried out three times, averaging the resulting sensitivities and noise standard deviations to compute the LODs shown in Table 1 as $\mathrm{LOD}=3 \sigma / \mathrm{S}$. Considering the highly distinct spectra of the two sources [Fig. 1(a)], it is remarkable that both extracted LODs are on the same order of magnitude, differing just by a factor of $\approx 2-3$ at the same power level. The ECL experiences a LOD improvement by a factor of $\approx 6$ when the input power is switched from low to high, the FPL a factor of $\approx 2$. The fact that they do not produce the same LOD is attributed mainly to the fact that the low-cost FPL is poorly temperature controlled. This can result in a drift of the central wavelength, which, combined with the wavelength response of the grating couplers, can introduce an unwanted modulation.

To validate the performance of the device as a biosensor, benchmark CRP protein detection experiments were performed with the high power setting. CRP was chosen because it is a widely used biomarker for inflammatory conditions [38]. Prior to each experiment, the following already successfully established offline chemical procedure was carried out [39]: the sensor chip surface was first oxidized by immersion in piranha solution, and subsequently amine-functionalized with (3-aminopropyl)trimethoxysilane (APTMS). Finally, anti-CRP antibodies were covalently attached to the surface via an amine-to-amine linker, bis(sulfosuccinimidyl)suberate (BS3). Before each detection experiment, the sensor surface was blocked with bovine serum albumin (BSA, $10 \mathrm{mg} / \mathrm{mL}$ ), to suppress non-specific binding. A flow rate of $20 \mu \mathrm{L} / \mathrm{min}$ was established for all CRP additive immunosensing experiments with a phosphate-buffered saline (PBS) $10 \mathrm{mM}$ (pH 7.4) as running buffer. Seven different CRP concentrations were subsequently injected to estimate the LOD: 10, 50, 200, 500, $1000,2000,4000 \mathrm{ng} / \mathrm{mL}$. Prior to that, BSA was injected at a concentration of $4000 \mathrm{ng} / \mathrm{mL}$ to confirm specificity. Figure 3 shows the sensors phase response according to the injected CRP concentration using the low-cost FPL. The BSA control injection causes a negligible phase shift, indicating the CRP specificity of the immunosensor surface. The phase shifts due to the CRP injections with concentrations of 10, 50, and

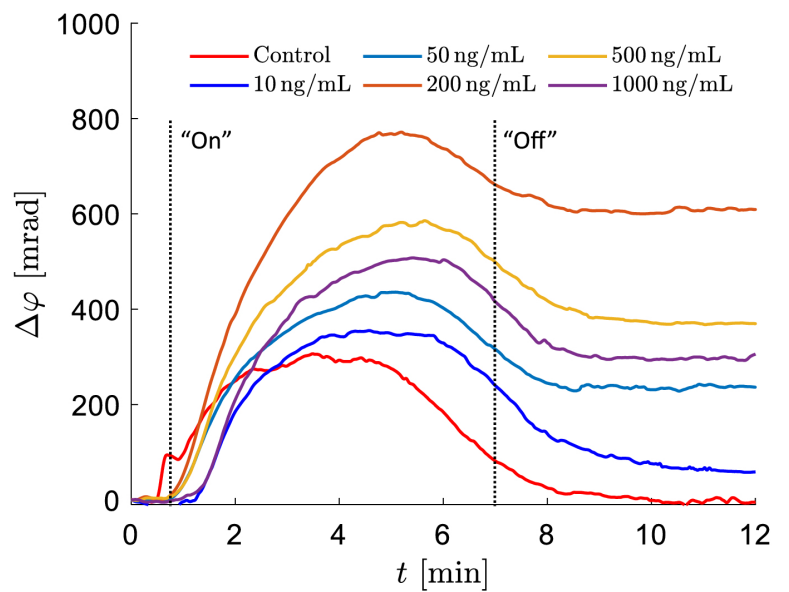

Fig. 3. Steady-state equilibrium phase shifts due to additive CRP immunoreactions using the FPL. The negligible shift of the control (BSA with a concentration of $4000 \mathrm{ng} / \mathrm{mL}$ ) is shown in red. For reasons of visibility, the two highest CRP injections are not shown. "On" and "Off" indicate the estimated start and end of the injections. The actual injection exposure time is likely longer due to diffusion within the fluidic system.

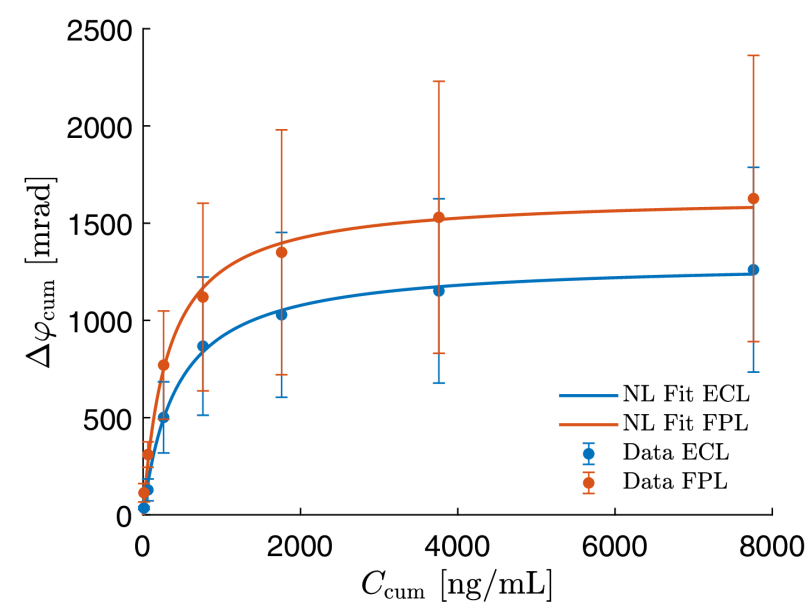

Fig. 4. CRP calibration curve obtained for triplicate immunosensing experiments with ECL and FPL, operated at higher power level. The data shown is the mean (filled circle) and standard deviation (error bars). The lines represent a nonlinear fit to the mean data.

$200 \mathrm{ng} / \mathrm{mL}$ follow a linear trend. However, as CRP accumulates on the surface, the biorecognition layer starts to saturate, and increasing concentrations yield smaller phase shifts. The corresponding mean calibration curves for CRP detection, for both the low-cost FPL and the reference ECL source, are represented in Fig. 4. The data of the cumulative introduced phase changes $\Delta \varphi_{\text {cum }}$ for each experiment were fitted to the function $B C_{\text {cum }} /\left(D+C_{\text {cum }}\right)$ achieving an excellent fitting ( $R^{2}>0.99$ for each calibration), with $C_{\text {cum }}$ the cumulative sum of the injected CRP concentrations, and $B, D$ the fitting parameters. The dots represent the mean cumulative phase shift and the error bars the standard deviation for the calibration curve for the corresponding cumulative concentration. The high standard deviation of the sensitivity can be explained by two factors. First, for each experiment, a different chip was used, each of which had been functionalized several times before, 
possibly degenerating the antibody immobilization efficiency. Second, but more importantly, our functionalization strategy requires further optimization to achieve reliable homogeneous surface coverage. A worst case sensitivity of $2 \mathrm{mrad} /(\mathrm{ng} / \mathrm{mL})$ and detection limit just below $300 \mathrm{pg} / \mathrm{mL}$ were extracted from the data with the reference ECL source. The mean sensitivity was $S=3.1 \mathrm{mrad} /(\mathrm{ng} / \mathrm{mL})$ with a LOD of $184 \mathrm{pg} / \mathrm{mL}$. This is significant improvement compared to previous CRP detection assays reported in the literature $[16,18,26]$ and highlights the potential of the coherent sensing approach. Using the low-cost FPL source, a sensitivity $S=5.2 \mathrm{mrad} /(\mathrm{ng} / \mathrm{mL})$ and an LOD of $1.9 \mathrm{ng} / \mathrm{mL}$ were observed. This moderate degradation is attributed mainly to the thermal instability of the source.

In this work, we have demonstrated the potential of coherently detected balanced MZIs for biosensing. We have demonstrated competitive detection limits, below 300 pg/mL for CRP, with a reference high performance optical source. Using a low-cost FPL, we were still able to achieve LODs below $2 \mathrm{ng} / \mathrm{mL}$ for CRP. We believe that these developments are an important step towards portable, low-cost, POC biosensor devices.

Funding. Horizon 2020 Framework Programme (EuroNanoMed 3-H2020 DrNanoDAII); Ministerio de Economía y Competitividad (2019/PCI 2019-2, CTQ2017-86994-R, SEV-2017-0706); Junta de Andalucía, Proyectos Excelencia-Retos (P18-RT-1453, P18-RT-793); Fondos Europeos de Desarrollo Regional (CTQ2016-75870-P, PID2019-104293GBI00, RD16/0006/0012, TEC2016-80718-R, UMA18-FEDERJA-007, UMA18-FEDERJA-219); H2020 Marie Skłodowska-Curie Actions (713721).

Disclosures. The authors declare no conflicts of interest.

\section{REFERENCES}

1. M. C. Cardenosa-Rubio, H. M. Robison, and R. C. Bailey, Curr. Opin. Environ. Sci. Heal. 10, 38 (2019).

2. P. Ramirez-Priego, D. Martens, A. A. Elamin, P. Soetaert, W. V. Roy, R. Vos, B. Anton, R. Bockstaele, H. Becker, M. Singh, P. Bienstman, and L. M. Lechuga, ACS Sens. 3, 2079 (2018).

3. A. F. Gavela, D. G. García, J. Ramirez, and L. Lechuga, Sensors 16, 285 (2016).

4. R. Baets, A. Z. Subramanian, S. Clemmen, B. Kuyken, P. Bienstman, N. L. Thomas, G. Roelkens, D. V. Thourhout, P. Helin, and S. Severi, in Optical Fiber Communication Conference (Optical Society of America, 2016), pp. 1-3.

5. B. Chocarro-Ruiz, A. Fernández-Gavela, S. Herranz, and L. M. Lechuga, Curr. Opin. Biotechnol. 45, 175 (2017).

6. J. G. Wangüemert-Pérez, A. Hadij-ElHouati, A. Sánchez-Postigo, J. Leuermann, D.-X. Xu, P. Cheben, A. Ortega-Moñux, R. Halir, and Í. Molina-Fernández, Opt. Laser Technol. 109, 437 (2019).

7. P. Cheben, R. Halir, J. H. Schmid, H. A. Atwater, and D. R. Smith, Nature 560, 565 (2018).

8. D. M. Kita, J. Michon, S. G. Johnson, and J. Hu, Optica 5, 1046 (2018).

9. M. Iqbal, M. A. Gleeson, B. Spaugh, F. Tybor, W. G. Gunn, M. Hochberg, T. Baehr-Jones, R. C. Bailey, and L. C. Gunn, IEEE J. Sel. Top. Quantum Electron. 16, 654 (2010).
10. R. J. J. van Gulik, B. M. de Boer, and P. J. Harmsma, IEEE J. Sel. Top. Quantum Electron. 23, 433 (2017).

11. J. Flueckiger, S. Schmidt, V. Donzella, A. Sherwali, D. M. Ratner, L. Chrostowski, and K. C. Cheung, Opt. Express 24, 15672 (2016).

12. S. Hu, Y. Zhao, K. Qin, S. T. Retterer, I. I. Kravchenko, and S. M. Weiss, ACS Photon. 1, 590 (2014).

13. Q. Liu, X. Tu, K. W. Kim, J. S. Kee, Y. Shin, K. Han, Y.-J. Yoon, G.-Q. Lo, and M. K. Park, Sens. Actuators B Chem. 188, 681 (2013).

14. A. D. Falco, L. O'Faolain, and T. F. Krauss, Appl. Phys. Lett. 94, 063503 (2009).

15. T. Claes, W. Bogaerts, and P. Bienstman, Proc. SPIE 8099, 80990R (2011).

16. E. Luan, H. Shoman, D. Ratner, K. Cheung, and L. Chrostowski, Sensors 18, 3519 (2018).

17. D. Martens and P. Bienstman, Nanophotonics 6, 703 (2017).

18. M. S. Luchansky, A. L. Washburn, M. S. McClellan, and R. C. Bailey, Lab Chip 11, 2042 (2011).

19. D. Martens, P. Ramirez-Priego, M. S. Murib, A. A. Elamin, A. B. Gonzalez-Guerrero, M. Stehr, F. Jonas, B. Anton, N. Hlawatsch, P. Soetaert, R. Vos, A. Stassen, S. Severi, W. V. Roy, R. Bockstaele, H. Becker, M. Singh, L. M. Lechuga, and P. Bienstman, Anal. Methods 10, 3066 (2018).

20. K. Schmitt, B. Schirmer, C. Hoffmann, A. Brandenburg, and P. Meyrueis, Biosens. Bioelectron. 22, 2591 (2007).

21. S. Dante, Opt. Pura y Apl. 45, 87 (2012).

22. K. Zinoviev, L. G. Carrascosa, J. S. del Río, B. Sepúlveda, C. Domínguez, and L. M. Lechuga, Adv. Opt. Technol. 2008, 1 (2008).

23. N. Skivesen, A. Têtu, M. Kristensen, J. Kjems, L. H. Frandsen, and P. I. Borel, Opt. Express 15, 3169 (2007).

24. J. Leuermann, A. Fernández-Gavela, A. Torres-Cubillo, S. Postigo, A. Sánchez-Postigo, L. M. Lechuga, R. Halir, and Í. Molina-Fernández, Sensors 19, 3671 (2019).

25. A. Densmore, D.-X. Xu, S. Janz, P. Waldron, T. Mischki, G. Lopinski, A. Delâge, J. Lapointe, P. Cheben, B. Lamontagne, and J. H. Schmid, Opt. Lett. 33, 596 (2008).

26. A. Psarouli, A. Botsialas, A. Salapatas, G. Stefanitsis, D. Nikita, G. Jobst, N. Chaniotakis, D. Goustouridis, E. Makarona, P. S. Petrou, I. Raptis, K. Misiakos, and S. E. Kakabakos, Talanta 165, 458 (2017).

27. S. Dante, D. Duval, B. Sepúlveda, A. B. González-Guerrero, J. R Sendra, and L. M. Lechuga, Opt. Express 20, 7195 (2012).

28. A. B. González-Guerrero, J. Maldonado, S. Herranz, and L. M. Lechuga, Anal. Methods 8, 8380 (2016).

29. Y. E. Marin, V. Toccafondo, P. Velha, Y. Jeong, S. Scarano, A. Nottola, S. Tirelli, H. P. Jeon, M. E. Minunni, F. D. Pasquale, and C. J. Oton, Proc. SPIE 10510, 1051005 (2018).

30. B. Luff, J. Wilkinson, J. Piehler, U. Hollenbach, J. Ingenhoff, and N. Fabricius, J. Lightwave Technol. 16, 583 (1998).

31. R. Halir, L. Vivien, X. L. Roux, D.-X. Xu, and P. Cheben, IEEE Photon. J. 5, 6800906 (2013).

32. J. Milvich, D. Kohler, W. Freude, and C. Koos, in IEEE Photonics Conference (IPC) (IEEE, 2017).

33. M. Knoerzer, C. Szydzik, G. Ren, C. S. Huertas, S. Palmer, P. Tang, T. G. Nguyen, L. Bui, A. Boes, and A. Mitchell, Opt. Express 27, 21532 (2019).

34. İ. Molina-Fernández, J. Leuermann, A. Ortega-Moñux, J. G. Wangüemert-Pérez, and R. Halir, Opt. Express 27, 12616 (2019).

35. P. J. Reyes-Iglesias, I. Molina-Fernández, A. Moscoso-Mártir, and A. Ortega-Moñux, Opt. Express 20, 5725 (2012).

36. VLC Photonics. Available online (https://www.vlcphotonics.com/).

37. J. E. Saunders, C. Sanders, H. Chen, and H.-P. Loock, Appl. Opt. 55, 947 (2016).

38. N. R. Sproston and J. J. Ashworth, Front. Immunol. 9, 754 (2018).

39. H. M. Robison and R. C. Bailey, Curr. Protoc. Chem. Biol. 9, 158 (2017). 